(c) 2016 IEEE. Personal use of this material is permitted. Permission from IEEE must be obtained for all other uses, in any current or future media, including reprinting/republishing this material for advertising or promotional purposes, creating new collective works, for resale or redistribution to servers or lists, or reuse of any copyrighted component of this work in other works 


\title{
Economic MPC with Periodic Terminal Constraints of Nonlinear Differential-Algebraic-Equation Systems: Application to Drinking Water Networks
}

\author{
Ye Wang, Vicenç Puig and Gabriela Cembrano
}

\begin{abstract}
In this paper, an Economic Model Predictive Control (EMPC) strategy with periodic terminal constraints is addressed for nonlinear differential-algebraic-equation systems with an application to Drinking Water Networks (DWNs). DWNs have some periodic behaviours because of the daily seasonality of water demands and electrical energy price. The periodic terminal constraint and economic terminal cost are implemented in the EMPC controller design for the purpose of achieving convergence. The feasibility of the proposed EMPC strategy when disturbances are considered is guaranteed by means of soft constraints implemented by using slack variables. Finally, the comparison results in a case study of the D-Town water network is provided by applying the EMPC strategy with or without periodic terminal constraints.
\end{abstract}

\section{INTRODUCTION}

Nowadays, Economic Model Predictive Control (EMPC) is one of the hot topics in the academic domain of Model Predictive Control (MPC). Recent developments have been reported in [1]. Reported results have proved that MPC is a powerful control strategy in many process industries in comparison with other available methods of multi-variable control. Unlike the classic MPC, the stage cost of the EMPC strategy may be non-convex and not directly related with the system states or outputs. Hence, the convergence and stability of the EMPC strategy may not be easy to achieve, since there is no reference trajectory for tracking.

For the operational management of Drinking Water Networks (DWNs), MPC has been discussed and adopted as a standard approach since it is able to maintain multi-objective operational goals and find optimal setpoints for the actuators (see [2], [3], [4], [5], [6] and [7]). In these references, the flow-based model that involves linear differential-algebraic equations has been considered as the control-oriented model and the EMPC strategy for DWNs has been applied by solving a finite-horizon optimization problem.

In [8] and [9], analysis of the asymptotic stability and average performance have been discussed for the EMPC strategy by adding terminal state constraints and results show that the average performance of the EMPC strategy is never worse than the optimal steady-state operation. The use of the terminal state constraints aims to force the system

Y. Wang, V. Puig and G. Cembrano are with Advanced Control Systems (SAC) research group at Institut de Robòtica i Informàtica Industrial, CSICUPC, Universitat Politècnica de Catalunya-BarcelonaTech, C/. Llorens i Artigas 4-6. 08028 Barcelona, Spain. E-mail: \{ywang, vpuig, cembrano\}eiri.upc. edu

G. Cembrano is also with CETaqua, Water Technology Centre, Ctra d'Esplugues 75, Cornellà de Llobregat, 08940 Barcelona, Spain. convergence and subsequently to achieve the stability. In [10] and [11], the EMPC strategy with periodic operation is introduced for the flow-based model of DWN. With the periodic terminal constraints, the asymptotic stability, as well as the average performance, can be achieved.

In many DWNs, the inclusion of pressure/head model is important in order to consider nonlinear constraints between flows and pressures/heads at some given nodes of the DWN as for example in demand sectors. The static relations of the head at each element in the DWN can be formulated with some algebraic variables by means of a relationship formula, for example the Hazen-Williams formula. As other process models, the resultant optimization problem involves complex systems of nonlinear differential-algebraic equations as presented in [12]. From the control point of view, the controloriented model of the DWN can be formulated as nonlinear differential-algebraic equations including pressure/head equations.

This paper proposes an EMPC strategy with periodic terminal state constraints for the nonlinear differentialalgebraic-equation model of the DWNs. The usual choice of the MPC prediction horizon for DWNs is 24 hours. However, periodic terminal state constraints need to be verified by using longer prediction horizons. Furthermore, the system disturbances have significant influences on the performance of the control system. Therefore, the periodic terminal constraints are designed considering the uncertain case. The idea to deal with the system disturbances in this paper is to use soft constraints to reconfigure periodic terminal state constraints. The proposed EMPC strategy has been tested in a case study based on the D-Town water network, using the EPANET hydraulic simulator. Comparison results of applied EMPC strategy with or without terminal constraint are shown.

The remainder of this paper is organized as follows. In Section II, the problem statement of the EMPC strategy with periodic terminal constraint is described and the strategy for dealing with uncertainty is discussed. In Section III, the EMPC strategy with periodic terminal constraints for the DWN is introduced. The case study of the D-Town water network is applied in order to test and compare the EMPC strategy with or without periodic terminal constraint and simulation results are shown in Section IV. Finally, some conclusions are drawn in Section V. 


\section{Problem Statement}

The generalized discrete-time differential-algebraic equations of a complex nonlinear system can be formulated as

$$
\begin{aligned}
& x_{k+1}=f\left(x_{k}, z_{k}, u_{k}, v_{k}, \omega_{k}\right), \\
& g\left(x_{k}, z_{k}, u_{k}, v_{k}, \omega_{k}\right)=0,
\end{aligned}
$$

where $x_{k} \in \mathbb{R}^{n_{x}}$ and $z_{k} \in \mathbb{R}^{n_{z}}$ denote the differential and algebraic system states at time instant $k \in \mathbb{N}$, respectively. $u_{k} \in \mathbb{R}^{n_{u}}$ and $v_{k} \in \mathbb{R}^{n_{v}}$ denote manipulated and nonmanipulated inputs at time instant $k$, respectively. $f(\cdot)$ and $g(\cdot)$ are linear or nonlinear mapping functions describing system dynamics and static relations, respectively. Moreover, $\omega_{k} \in \mathbb{R}^{n_{w}}$ represents the measured system disturbances at time instant $k$, which is assumed to be composed of two parts: deterministic disturbance $\omega_{k}^{d}$ and stochastic disturbance $\omega_{k}^{s}$, and can be formulated as

$$
\omega_{k}=\omega_{k}^{d}+\omega_{k}^{s}
$$

Assumption 1: The deterministic disturbance $\omega_{k}^{d}$ is assumed as known (typically predicted using a forecasting model) and bounded input for the system (1) while the stochastic disturbance $\omega_{k}^{s}$ represents the noise with zeromean and small-variance $\sigma_{s}^{2}$ characteristics that can be described by means of a Gaussian model as $\omega_{k}^{s} \sim \mathcal{N}\left(0, \omega_{k}^{s}\right)$.

Considering Assumption 1, the differential-algebraic equations in (1) can be reformulated as

$$
\begin{aligned}
& x_{k+1}=f\left(x_{k}, z_{k}, u_{k}, v_{k}, \omega_{k}^{d}\right)+\epsilon\left(\omega_{k}^{s}\right), \\
& g\left(x_{k}, z_{k}, u_{k}, v_{k}, \omega_{k}^{d}\right)+\eta\left(\omega_{k}^{s}\right)=0,
\end{aligned}
$$

where $\epsilon(\cdot)$ and $\eta(\cdot)$ are disturbance-transfer functions for system dynamics and static relations, respectively.

Definition 1: The described system in (3) is called $T$ periodic system if there exists a minimal non-zero $T \in \mathbb{N}$ so that the following conditions hold

$$
\begin{aligned}
& f\left(x_{k}, z_{k}, u_{k}, v_{k}, \omega_{k}^{d}\right)=f\left(x_{k+n T}, z_{k+n T}, u_{k+n T}, v_{k+n T}, \omega_{k+n T}^{d}\right) \\
& g\left(x_{k}, z_{k}, u_{k}, v_{k}, \omega_{k}^{d}\right)=g\left(x_{k+n T}, z_{k+n T}, u_{k+n T}, v_{k+n T}, \omega_{k+n T}^{d}\right)
\end{aligned}
$$

and $T$ is called the period of the system (3).

Generally speaking, the system states including differential and algebraic variables, manipulated and non-manipulated control inputs in (3) are required to satisfy equality and inequality constraints in the form of a polyhedral set defined as

$$
\left(x_{k}, z_{k}, u_{k}, v_{k}\right) \in \mathbb{C}_{k}, \quad \forall k \in \mathbb{N}_{\geq 1}
$$

with

$$
\mathbb{C}_{k} \triangleq\left\{(x, z, u, v) \mid a_{k, i} x+b_{k, i} z+c_{k, i} u+d_{k, i} v \leq \gamma_{k, r}\right\},
$$

where $r \in \mathbb{N}_{\geq 1}$ is the number of hyperplanes and $\forall i \in \mathbb{N}_{[1, r]}$. $a_{k, i} \in \mathbb{R}^{1 \times \bar{n}_{x}}, b_{k, i} \in \mathbb{R}^{1 \times n_{z}}, c_{k, i} \in \mathbb{R}^{1 \times n_{u}}$ and $d_{k, i} \in$ $\mathbb{R}^{1 \times n_{v}}$ are the constraint vectors.

The non-convex cost function taking multiple controloriented objectives into account is given by

$$
\sum_{k} \ell\left(x_{k}, z_{k}, u_{k}, v_{k}, \omega_{k}^{d}\right) \text {. }
$$

Usually, MPC solves a finite optimization problem. However, the MPC prediction horizon can be ideally selected as infinite. For the $T$-periodic system in (3), a sufficiently long prediction horizon can be chosen including several periods. Therefore, as proposed in [8], Equation (7) can be formulated in average form as follows

$$
V_{n T}\left(x_{k}, \mathbf{u}\right) \triangleq \sum_{i=0}^{n T-1} \frac{\ell\left(x_{k+i \mid k}, z_{k+i \mid k}, u_{k+i \mid k}, v_{k+i \mid k}, \omega_{k+i \mid k}^{d}\right)}{n T},
$$

where $\mathbf{u}=\left[u_{0}, u_{1}, \ldots, u_{n T-1}\right]^{T}$ and $n \in \mathbb{N}_{\geq 1}$.

The EMPC strategy for the periodic system can be implicitly formulated by solving the following optimization problem:

$$
\min _{\mathbf{u}^{*}} V_{n T}\left(x_{k}, \mathbf{u}\right),
$$

subject to

$$
\begin{aligned}
& x_{k+i+1 \mid k}=f\left(x_{k+i \mid k}, z_{k+i \mid k}, u_{k+i \mid k}, v_{k+i \mid k}, \omega_{k+i \mid k}^{d}\right), \\
& g\left(x_{k+i \mid k}, z_{k+i \mid k}, u_{k+i \mid k}, v_{k+i \mid k}, \omega_{k+i \mid k}^{d}\right)=0, \\
& \left(x_{k+i \mid k}, z_{k+i \mid k}, u_{k+i \mid k}, v_{k+i \mid k}, \omega_{k+i \mid k}^{d}\right) \in \mathbb{C}_{k+i \mid k}, \\
& x_{k+n T \mid k}=x_{k \mid k}, \\
& \left(x_{k \mid k}, \omega_{k \mid k}^{d}\right)=\left(x_{k}, \omega_{k}\right),
\end{aligned}
$$

where the predicted system states can be calculated in (9b) by means of the nominal part of (3) assuming that deterministic disturbances $\omega_{k}^{d}$ can be forecasted along the MPC prediction horizon. (9e) denotes the terminal constraint for the periodic system. (9f) denotes the measurements of last system states and disturbances as the initial condition of the MPC controller. If the aforementioned problem is feasible, the admissible set for the pair of $\left(x_{k}, \mathbf{u}\right)$ can be defined as $\mathbb{Y}_{n T}$ such that the set of admissible states $\mathcal{X}_{n T}$ can be defined by the projection of $\mathbb{Y}_{n T}$ to $\mathbb{X}$ as follows:

$$
\mathcal{X}_{n T}=\left\{x_{k} \in \mathbb{X} \mid \forall \mathbf{u},\left(x_{k}, \mathbf{u}\right) \in \mathbb{Y}_{n T}\right\} .
$$

The terminal state constraint has been set in (9e), which enforces the terminal states to be equal to the initial state. If the system is following the mathematical model (3), the initial state is affected by the stochastic disturbance $\omega^{s}$ and differ from the nominal system model in a narrow interval so that the optimization problem could be infeasible at a certain time instant. In order to avoid the infeasibility and maintain the terminal state constraint, the terminal region $\mathbb{X}_{f}$ is adopted, which can be denoted by means of a closed circle of radius defined by a slack variable $\delta_{k} \in \mathbb{R}_{+}$and centered at the initial state $x_{k}$ :

$$
\mathcal{X}_{n T} \supseteq \mathbb{X}_{f}\left(x_{k}, \delta_{k}\right) \triangleq\left\{x \mid\left\|x_{k+n T}-x_{k}\right\| \leq \delta_{k}, \delta_{k} \geq 0\right\},
$$

where $\|\cdot\|$ denotes the 2-norm operator. The terminal cost aims to minimize the radius $\delta$ that is a vector of slack variables for each system state. Therefore, the terminal cost function can be written as

$$
V_{f}\left(x_{k+n T \mid k}, \delta_{k}\right)=\left\|\delta_{k}\right\|^{2} .
$$

The economic cost stage function can be implemented as follows:

$$
\widetilde{V}_{n T}\left(x_{k}, \mathbf{u}\right) \triangleq V_{n T}\left(x_{k}, \mathbf{u}\right)+V_{f}\left(x_{k+n T \mid k}, \delta_{k}\right) .
$$


Thus, the optimization problem can be reformulated as

$\min _{\mathbf{u}^{*}, \delta^{*}} \widetilde{V}_{n T}\left(x_{k}, \mathbf{u}, \delta_{k}\right)$,

subject to

$$
\begin{aligned}
& x_{k+i+1 \mid k}=f\left(x_{k+i \mid k}, z_{k+i \mid k}, u_{k+i \mid k}, v_{k+i \mid k}, \omega_{k+i \mid k}^{d}\right)+\epsilon\left(\omega_{k+i \mid k}^{s}\right), \\
& g\left(x_{k+i \mid k}, z_{k+i \mid k}, u_{k+i \mid k}, v_{k+i \mid k}, \omega_{k+i \mid k}^{d}\right)+\eta\left(\omega_{k+i \mid k}^{s}\right)=0, \\
& \left(x_{k+i \mid k}, u_{k+i \mid k}, \omega_{k+i \mid k}^{d}\right) \in \mathbb{C}_{k+i \mid k}, \\
& x_{k+n T \mid k} \in \mathbb{X}_{f}\left(x_{k \mid k}, \delta_{k}\right) \\
& \left(x_{k \mid k}, \omega_{k \mid k}^{d}\right)=\left(x_{k}, \omega_{k}\right) .
\end{aligned}
$$

\section{ECONOMIC MOdel Predictive CONTROL FOR DRINKING WATER NETWORKS}

\section{A. The Control-oriented Model of DWNs}

The control-oriented model of DWNs is built by using discrete-time differential-algebraic equations (1) that can be written in the following form at the time instant $k \in \mathbb{N}$ [13]:

$$
\begin{aligned}
& x_{k+1}=A x_{k}+B_{u} u_{k}+B_{v} v_{k}+B_{d} d_{k}, \\
& E_{u} u_{k}+E_{v} v_{k}+E_{d} d_{k}=0, \\
& P_{x} x_{k}+P_{z} z_{k}=\psi\left(u_{k}, v_{k}\right),
\end{aligned}
$$

where $x_{k} \in \mathbb{R}^{n_{x}}$ denotes the heads at the storage nodes (tanks) corresponding to the differential state vector. $z_{k} \in$ $\mathbb{R}^{n_{z}}$ denotes the heads at the non-storage nodes, which which corresponds to the algebraic state vector. $u_{k} \in \mathbb{R}^{n_{u}}$ denotes manipulated flow rates of the actuators (pumps and valves). $v_{k} \in \mathbb{R}^{n_{v}}$ denotes non-manipulated flow rates through pipes. $d_{k} \in \mathbb{R}^{n_{d}}$ denotes the water demands, which are regarded as the measured system disturbances. (15a) describes the system dynamics, (15b) describes the physical and static relations in the water network by means of mass balance at non-storage nodes and (15c) describes head-flow relationship by means of the Hazen-Williams formula. Furthermore, $A, B_{u}, B_{v}, B_{d}, E_{u}, E_{v}, E_{d}, P_{x}$ and $P_{z}$ are timeinvariant matrices with suitable dimensions decided by the network topology while $\psi$ is a vector of the nonlinear mapping functions.

Assumption 2: The water demand $d_{k}$ is considered as the system disturbance $\omega_{k}$, which is described in (2). The deterministic water demand $\bar{d}_{k}$ follows a cyclic pattern with a period $T$. The stochastic demand $\Sigma_{d_{k}}$ is assumed as a noise signal with respect to zero-mean Gaussian distribution. Hence, the nominal water demand presents a periodic property (typically daily), where $\bar{d}_{k}=\bar{d}_{k+T}$ and then $d_{k} \approx d_{k+T}$. The stochastic demands are assumed to be predicted along the MPC prediction horizon by means of a suitable forecasting algorithm, for instance Gaussian process algorithm described in [14].

According to Assumption 2, Equation (15a) and (15b) can be adapted as follows:

$$
\begin{aligned}
& x_{k+1}=A x_{k}+B_{u} u_{k}+B_{v} v_{k}+B_{d} \hat{d}_{k}, \\
& E_{u} u_{k}+E_{v} v_{k}+E_{d} \hat{d}_{k}=0,
\end{aligned}
$$

where

$$
\hat{d}_{k}=\bar{d}_{k}+\Sigma_{d_{k}}
$$

\section{B. Constraint Settings}

The system states and some control inputs including the manipulated and non-manipulated variables described in (15) are subject to physical limitations, which can be characterized for all $k \in \mathbb{N}_{+}$as

$$
\begin{aligned}
& x_{\min } \leq x_{k} \leq x_{\max } \\
& z_{\min } \leq z_{k} \leq z_{\max }, \\
& u_{\min } \leq u_{k} \leq u_{\max } \\
& v_{\min } \leq v_{k} \leq v_{\max }
\end{aligned}
$$

where $x_{\min }, x_{\max } \in \mathbb{R}^{n_{x}}$ represents the maximal and minimal head at the storage tanks that is subject to the physical capacity of the tanks, respectively. $z_{\min }, z_{\max }$ represents the minimal and maximal required heads for some nonstorage nodes. $u_{\min }, u_{\max } \in \mathbb{R}^{n_{u}}$ represents the minimal and maximal flow rates for actuators, respectively. $v_{\min }, v_{\max }$ represents the minimal and maximal required flow rates through certain interconnected pipes.

Remark 1: In terms of the demand sectors, $z_{\max }$ is not mandatory and $z_{\min }$ is required to guarantee the adequate level of service to the customers. In terms of interconnected pipes, $v_{\min }$ and $v_{\max }$ are alternative and not mandatory.

Taking the system safety and reliability into account, the system states $x_{k}$ must be guaranteed to be above reserved safety heads for all $k \in \mathbb{N}$ by using the following soft constraint:

$$
\begin{aligned}
& x_{k} \geq \pi-\zeta_{k}, \\
& \zeta_{k} \geq 0,
\end{aligned}
$$

where $\pi \in \mathbb{R}^{n_{x}}$ denotes a vector of the minimal heads of the storage tanks for the sake of satisfying the underlying demanded water from tanks and maintaining the safety heads and $\zeta_{k} \in \mathbb{R}^{n_{x}}$ represents a vector of slack variables to be minimized, which is used for relaxing the stored water below the safety head as a soft constraint.

Besides, the terminal region mentioned in (11) is adopted as an additional constraint for management of the DWN. The radius $\delta_{k}$ is a vector of slack variable only related to the terminal state at time instant $k$ to be minimized.

\section{DWN Management Criteria}

The common management criteria for the DWN can be found in [4], [6] and [5], which include economic, safety and smoothness terms. The cost functions corresponding to these control-oriented objectives are formulated with standard quadratic forms as follows:

$$
\begin{aligned}
& \ell_{k}^{e} \triangleq \alpha_{k}^{\prime} \Phi_{e} u_{k}, \\
& \ell_{k}^{m} \triangleq \zeta_{k}^{\prime} \Phi_{m} \zeta_{k}, \\
& \ell_{k}^{r} \triangleq \Delta u_{k}^{\prime} \Phi_{r} \Delta u_{k}, \\
& \ell^{t} \triangleq \delta_{k}^{\prime} \Phi_{p} \delta_{k},
\end{aligned}
$$

with

$$
\begin{gathered}
\alpha_{k} \triangleq \alpha_{1}+\alpha_{2, k}, \\
\Delta u_{k} \triangleq u_{k}-u_{k-1},
\end{gathered}
$$

where (20a) denotes the economic costs of operating the whole network relying on assumed periodic energy price given by (21a), $\alpha_{1} \in \mathbb{R}^{n_{u}}$ is the fixed water production cost 
depending on the selected water source and $\alpha_{2} \in \mathbb{R}^{n_{u}}$ is the energy price depending on the dynamic electricity tariff. It is assumed that $\alpha_{k}$ has the same periodic characteristic as the system state, $\alpha_{k}=\alpha_{k+T}$. $\ell_{k}^{m}$ represents the safety cost by means of penalizing the slack variable found in (19). $\ell_{k}^{r}$ describes the smoothness cost that guarantees a smooth operation to maximize the lifetime of actuators in the network. $\ell^{t}$ draws the terminal cost that penalizes radius $\delta_{k}$ of the terminal region to find minimum, ideally equal to zero. Moreover, $\Phi_{e}, \Phi_{m}$ and $\Phi_{r}$ are weighting matrices for selecting corresponding variables with suitable dimensions.

Then, the multi-objective stage cost function and terminal cost function can be defined in a weighted form, respectively:

$$
\begin{aligned}
V_{s} & \triangleq \frac{1}{n T} \sum_{i=0}^{n T-1}\left\{\lambda_{1} \ell_{k+i \mid k}^{e}+\lambda_{2} \ell_{k+i \mid k}^{m}+\lambda_{3} \ell_{k+i \mid k}^{r}\right\}, \\
V_{f}\left(\delta_{k}\right) & \triangleq \lambda_{4} \ell^{t}
\end{aligned}
$$

where $\lambda_{1}, \lambda_{2}, \lambda_{3}, \lambda_{4}$ are prioritization weights that establish the importance of each related objective. Hence, the proposed EMPC strategy can be realized along $n T$ prediction horizon $(n \in \mathbb{N}$ ) by solving the following optimization problem:

$$
\min _{\mathbf{u}^{*}, \zeta^{*}, \delta^{*}} V_{s}+V_{f}\left(\delta_{k}\right),
$$

subject to

$$
\begin{aligned}
& x_{k+i+1 \mid k}=A x_{k+i \mid k}+B_{u} u_{k+i \mid k}+B_{v} v_{k+i \mid k}+B_{d} \hat{d}_{k+i \mid k} \\
& E_{u} u_{k+i \mid k}+E_{v} v_{k+i \mid k}+E_{d} \hat{d}_{k+i \mid k}=0 \\
& P_{x} x_{k+i \mid k}+P_{z} z_{k+i \mid k}=\psi\left(u_{k+i \mid k}, v_{k+i \mid k}\right) \\
& x_{\min } \leq x_{k+i \mid k} \leq x_{\max } \\
& z_{\min } \leq z_{k+i \mid k} \leq z_{\max } \\
& u_{\min } \leq u_{k+i \mid k} \leq u_{\max } \\
& v_{\min } \leq v_{k+i \mid k} \leq v_{\max } \\
& x_{k+i \mid k} \geq \pi-\zeta_{k+i} \\
& \left\|x_{k+n T \mid k}-x_{k \mid k}\right\| \leq \delta_{k} \\
& \left(x_{k \mid k}, \hat{d}_{k \mid k}\right)=\left(x_{k}, d_{k}\right)
\end{aligned}
$$

After the above-referred optimization problem is solved, a series of optimal control actions along the MPC prediction horizon can be obtained. Then, by means of the receding horizon approach, the first control action is applied to the real system at current time instant.

\section{Case Study: D-Town Water Network}

In order to illustrate and assess the proposed EMPC strategy, the D-Town water network is chosen as the case study. The benchmark of D-Town water network is composed of 388 nodes, 405 links, 7 tanks, and contains multiple unidirectional and bidirectional links. The aggregated model of the D-Town network shown in Fig. 1 is obtained by removing all the terminal links connecting to single demands and assigning the demands of the removed nodes to the appropriate root node. Similarly, a pressure constraint is added at the root node to guarantee that the head is sufficient to supply the demand with the maximum head required at the removed nodes. Therefore, the control-oriented model of the D-Town water network contains 7 states, 6 control inputs, 85 water demands, several hydraulic heads at nonstorage nodes and several non-manipulated flows through the interconnected pipes [13].

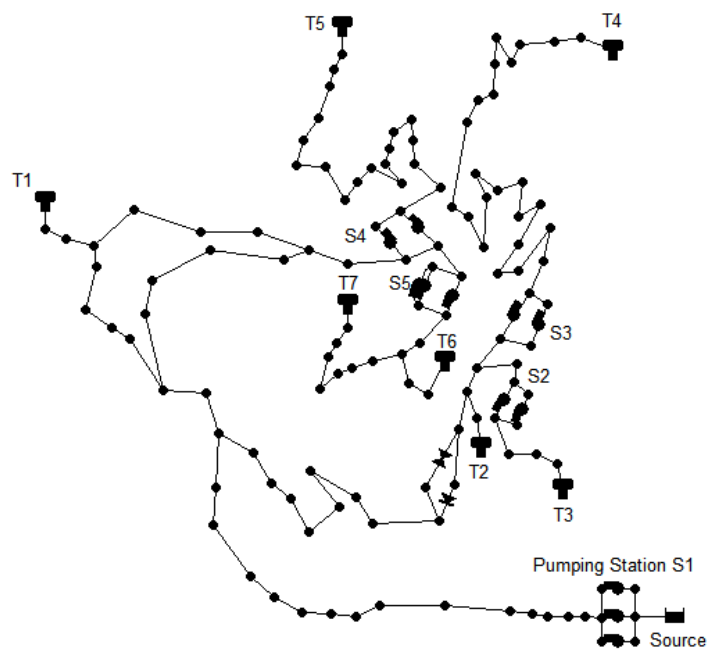

Fig. 1. Aggregated Topology of the D-Town Network

\section{A. Simulation Results}

The simulation with different EMPC settings have been executed in a PC of Intel Core i7-5500U CPU and 12GB RAM with MATLAB R2015a. The EMPC controller has been using GAMS (General Algebraic Modeling System) optimization modeling language, which is widely used for modeling large-scale and complex nonlinear optimization problem. The resultant nonlinear optimization problem is solved using the nonlinear solver CONOPT [15]. Fig. 2 shows the online simulation platform that is used in this case study, where MATLAB is used as the communication tool between the optimizer and EPANET simulator and a pump scheduling approach (see [13]) is implemented in the MATLAB. Besides, the data of predicted demands and electricity price are stored in the database.

Fig. 3 shows the collected data of water demands in 7 days, where it can be noticed that there exists a potential daily pattern with the assumption of two parts (deterministic and stochastic demands) that corresponds with the period of the system (15).

The sampling time is selected as 1 hour. The period of the D-Town network is chosen to be 24 hours. Hence, the MPC prediction horizon $H_{p}$ of 24 and 48 (hours) are chosen. The simulation results in 4 days are computed for the EMPC strategy with periodic terminal constraint in Problem (23). For comparison, the conventional EMPC strategy are used. The weights for the prioritization of the cost functions have been selected $\lambda_{1}=100, \lambda_{2}=10, \lambda_{3}=1$ and $\lambda_{4}=10$.

In order to compare the performance of the proposed EMPC strategy with different settings, the following key 


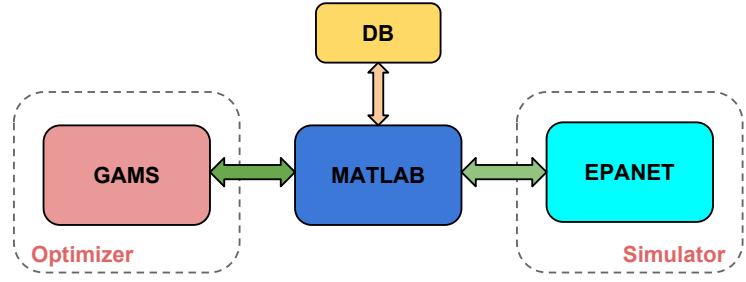

Fig. 2. Online Simulation Platform

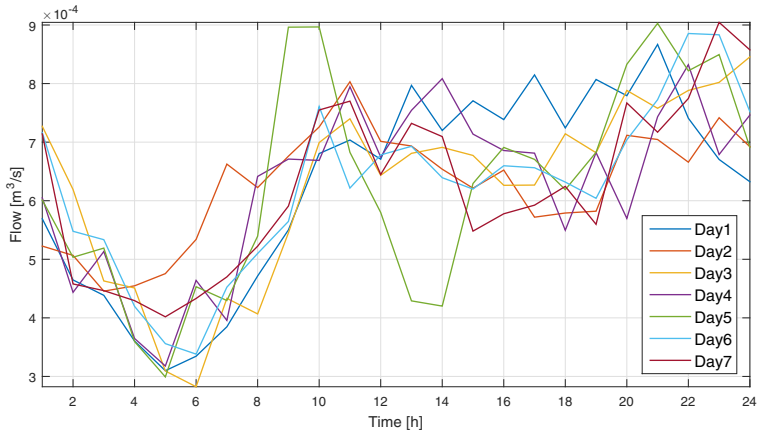

Fig. 3. The Water Demand Data sampled from the D-Town Network

performance indicators (KPIs) are used [6]:

$$
\begin{aligned}
K P I_{E} & \triangleq \frac{1}{n_{s}} \sum_{k=1}^{n_{s}} \alpha_{k}^{\prime} u_{k}, \\
K P I_{S} & \triangleq \sum_{k=1}^{n_{s}} \sum_{i=1}^{n_{x}} \max \left\{0,\left(\pi_{(i), k}-x_{(i), k}\right)\right\} \\
K P I_{M} & \triangleq \frac{1}{n_{s}} \sum_{k=1}^{n_{s}} \sum_{i=1}^{n_{x}}\left(x_{(i), k}-\pi_{(i), k}\right), \\
K P I_{\Delta U} & \triangleq \frac{1}{n_{s}} \sum_{k=1}^{n_{s}} \sum_{i=1}^{n_{u}}\left(\Delta u_{(i), k}\right)^{2}, \\
K P I_{T} & \triangleq \frac{1}{n_{s}} \sum_{k=1}^{n_{s}} \sum_{i=1}^{n_{x}}\left(\delta_{(i), k}\right)^{2},
\end{aligned}
$$

where $K P I_{E}$ denotes the economic KPI that evaluates the costs of the DWN management, $K P I_{S}$ considers safety KPI, which should be always 0 if the system is running safely. $K P I_{M}$ presents the measured safety KPI that accumulates average volume of remaining water in each storage tank, together with $K P I_{S}$ estimating the safety levels under different settings, and $K P I_{\Delta U}$ addresses smoothness KPI that computes the collected slew rates. $K P I_{T}$ denotes the terminal constraint KPI which computes the section of terminal region by means of the radius $\delta$. Moreover, $n_{s}$ represents the number of hours considered in the assessment.

The comparative simulation results of the D-Town water network are shown in Fig. 4 and Fig. 5. Fig. 4 shows the comparisons of heads at two selected storage tanks with the four conditions which corresponds to the following cases: the EMPC without terminal state constraint with the 24-hour prediction horizon, the EMPC with terminal state constraint with the 24-hour prediction horizon, the EMPC without terminal state constraint with the 48-hour prediction horizon

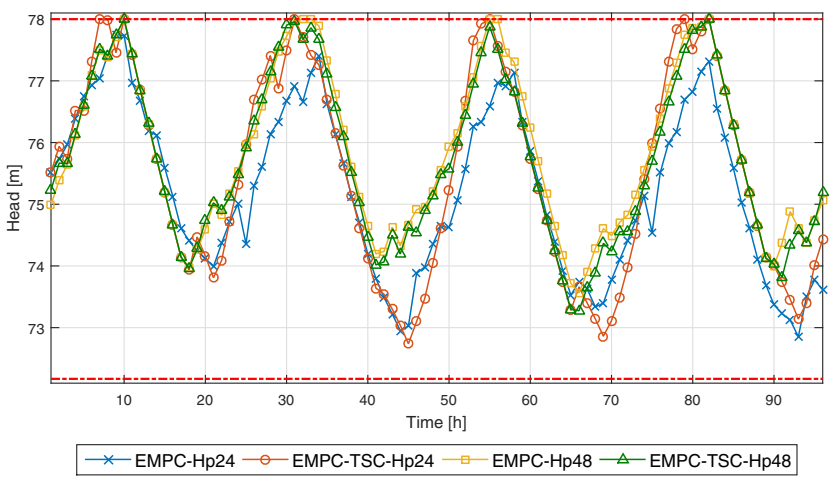

(a) Tank: T1

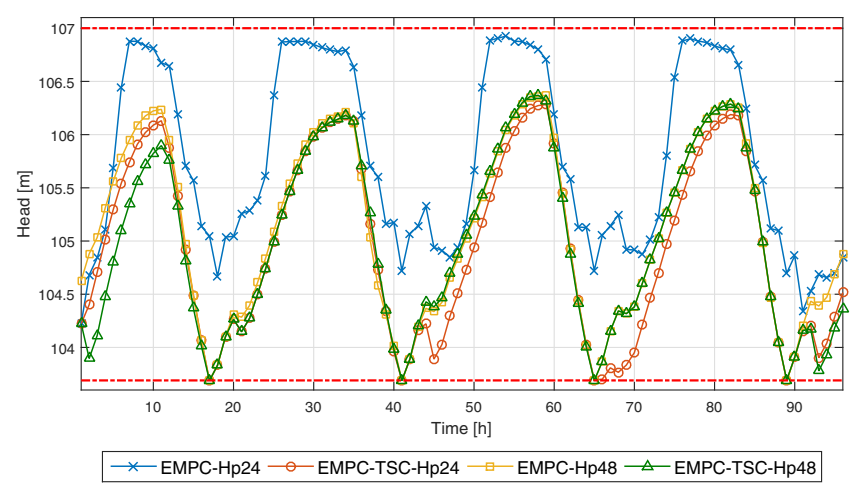

(b) Tank: T7

Fig. 4. Comparisons of Heads at the Storage Tank: T1 and T7

and the EMPC with terminal state constraint with the 48hour prediction horizon. For the tank T7 in Fig. 4(b), the state (blue line) with 24-hour horizon case is far away from the other cases while the other cases are approximately equivalent, which implies the less horizon case is not closed to be stable. When the prediction horizon is enlarged up to 48 hours, the head evolutions in all the tanks are changed because the satisfaction of the water demands in the extended horizon has been taken into account. Ideally, an infinite prediction horizon will bring the best performance of the considered control system. But an extended horizon increases the computation time of solving optimization problem including demand forecasting and the computation time of one step should be lower than the sampling time. Hence, a suitable prediction horizon is necessary taking all mentioned points into consideration. Besides, the simulation results of the EMPC with terminal state constraint are also shown. From Fig. 4(b), it is obvious that the head evolution with terminal constraint is close to the case with extended horizon. Comparison results of the water flows through pumping station and valve are shown in Fig. 5 and Fig. 6, respectively. The flows through pumping station $\mathrm{S} 2$ are selected when the electricity price is low. Hence, the EMPC strategy is effective to control the operating costs associated to of the DWN.

Assessment results provided by KPIs are shown in Table I. From the results in $K P I_{E}$, it may be observed that enlarging 


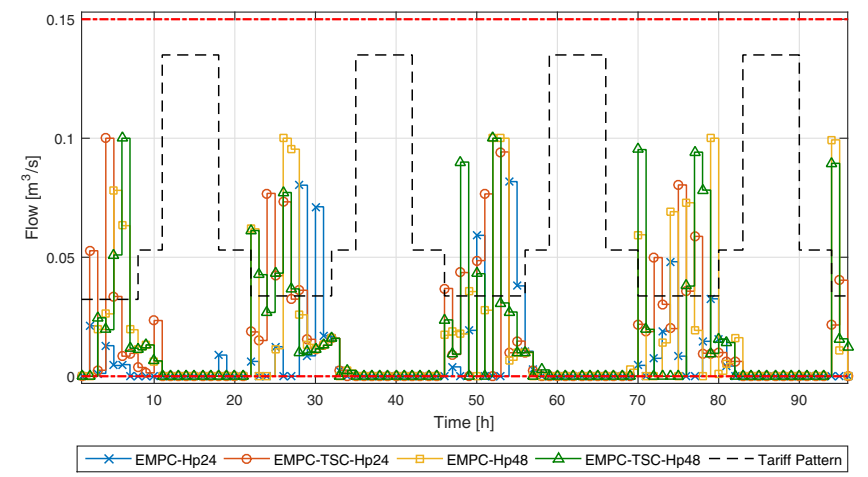

Fig. 5. Comparisons of Pumping Flow in Pumping Station: S2

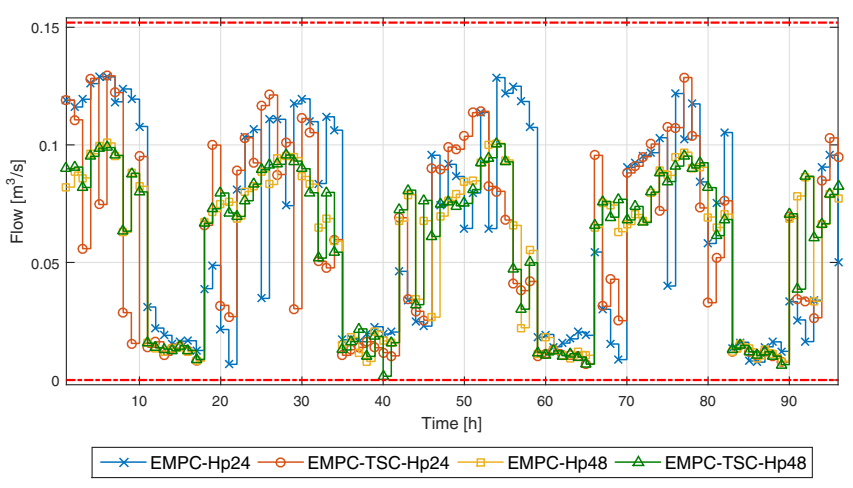

Fig. 6. Comparisons of Water Flow in Valve: V2

the prediction horizon $H_{p}$ brings more operational costs for the purpose of approaching convergence situation in terms of the system states. In terms of $K P I_{\Delta U}$, the performances of the cases with enlarged prediction horizon and periodic terminal constraints are better than the one with only 24-hour horizon and without terminal constraints. Besides, $K P I_{S}$, $K P I_{M}$ and $K P I_{T}$ have been omitted for all the different controllers in Table I since they are never below their safety levels and the optimal terminal cost can be zero when the optimization problem of the EMPC is solved by the selected nonlinear optimization solver.

\section{CONCLUSions}

In this paper, the EMPC strategy with periodic terminal state constraints has been investigated when applied to nonlinear differential-algebraic equation systems and an additional strategy for dealing with uncertainty has also been proposed. The EMPC strategy with periodic terminal state constraints has been subsequently applied to the complex D-Town water network and the online simulation results and comparisons with cases of enlarging MPC prediction horizon and ignoring the periodic terminal state constraints have shown its effectiveness. All the tests show similar results in terms of performance indexes, but the approach with terminal constraint is a step towards guaranteeing stability. Slightly larger values of the economic KPI when using longer prediction horizon are to be expected due to the handling of
TABLE I

COMPARISONS OF DifFEREnT EMPC CONTROLler Peformances

\begin{tabular}{ccc}
\hline Controller & $K P I_{E}$ & $K P I_{\Delta U}$ \\
\hline EMPC- $H_{p} 24$ & 23.5067 & 0.1195 \\
EMPC- $H_{p} 48$ & 26.0587 & 0.0807 \\
EMPC-TSC- $H_{p} 24$ & 25.0330 & 0.0962 \\
EMPC-TSC- $H_{p} 48$ & 26.0783 & 0.0811 \\
\hline
\end{tabular}

uncertainty along the prediction horizon. As further research, the proof of recursive feasibility and stability of the proposed approach will be addressed in a formal way.

\section{ACKNOWLEDGMENT}

This work has been supported by the Spanish project ECOCIS (DPI-2013-48243-C2-1-R). Ye Wang also thanks Consejo Superior de Investigaciones Científicas (CSIC) for providing FPI grant to support his PhD study.

\section{REFERENCES}

[1] D. Q. Mayne, "Model predictive control: Recent developments and future promise," Automatica, vol. 50, no. 12, pp. 2967 - 2986, 2014.

[2] G. Cembrano, G. Wells, J. Quevedo, R. Perez, and R. Argelaguet, "Optimal control of a water distribution network in a supervisory control system," Control Engineering Practice, vol. 8, no. 10, pp. 1177 $-1188,2000$.

[3] G. Cembrano, J. Quevedo, M. Salamero, V. Puig, J. Figueras, and J. Marti, "Optimal control of urban drainage systems: A case study," Control Engineering Practice, vol. 12, no. 1, pp. 1 - 9, 2004.

[4] C. Ocampo-Martinez, V. Puig, G. Cembrano, and J. Quevedo, "Application of MPC strategies to the management of complex networks of the urban water cycle," IEEE Control Systems Magazine, vol. 33, no. 1, pp. 15-41, 2013.

[5] J. M. Grosso, C. Ocampo-Martinez, V. Puig, and B. Joseph, "Chanceconstrained model predictive control for drinking water networks," Journal of Process Control, vol. 24, no. 5, pp. 504-516, 2014.

[6] Y. Wang, C. Ocampo-Martinez, and V. Puig, "Robust model predictive control based on gaussian processes: Application to drinking water networks," in European Control Conference, Linz, Austria, 2015.

[7] - "Stochastic model predictive control based on gaussian processes applied to drinking water networks," IET Control Theory \& Applications, 2016, in Press.

[8] D. Angeli, R. Amrit, and J. B. Rawlings, "On average performance and stability of economic model predictive control," IEEE Transactions on Automatic Control, vol. 57, no. 7, pp. 1615-1626, July 2012.

[9] M. Diehl, R. Amrit, and J. Rawlings, "A lyapunov function for economic optimizing model predictive control," IEEE Transactions on Automatic Control, vol. 56, no. 3, pp. 703-707, 2011.

[10] D. Limon, M. Pereira, D. Muñoz de la Peña, T. Alamo, and J. Grosso, "Single-layer economic model predictive control for periodic operation," Journal of Process Control, vol. 24, no. 8, pp. 1207 - 1224, 2014.

[11] J. M. Grosso, "On model predictive control for economic and robust operation of generalised flow-based networks," Ph.D. dissertation, Polytechnic University of Catalonia, 2015.

[12] M. Diehl, H. Bock, J. Schlöder, R. Findeisen, Z. Nagy, and F. Allgöwer, "Real-time optimization and nonlinear model predictive control of processes governed by differential-algebraic equations," Journal of Process Control, vol. 12, no. 4, pp. 577 - 585, 2002.

[13] Y. Wang, V. Puig, and G. Cembrano, "Nonlinear economic model predictive control of water distribution networks," Journal of Process Control, 2016, submitted.

[14] Y. Wang, C. Ocampo-Martinez, V. Puig, and J. Quevedo, "Gaussianprocess-based demand forecasting for predictive control of drinking water networks," in 9th International Conference on Critical Information Infrastructures Security, Limassol, Cyprus, 2014.

[15] A. Brooke, D. Kendrick, A. Meeraus, and R. Raman, GAMS, ser. A User's Guide. Washington DC, USA: GAMS Development Corporation, 2004. 